\title{
Parts of the Whole: Teaching Quantitative Reasoning in the Predator-Prey Model
}

Dorothy Wallace

Dartmouth College, dorothy.wallace@dartmouth.edu

Follow this and additional works at: https://digitalcommons.usf.edu/numeracy

Part of the Curriculum and Instruction Commons, Dynamic Systems Commons, Educational Methods Commons, Science and Mathematics Education Commons, and the Systems Biology Commons

\section{Recommended Citation}

Wallace, Dorothy. "Parts of the Whole: Teaching Quantitative Reasoning in the Predator-Prey Model." Numeracy 9, Iss. 1 (2016): Article 10. DOI: http://dx.doi.org/10.5038/1936-4660.9.1.10 


\title{
Parts of the Whole: Teaching Quantitative Reasoning in the Predator-Prey Model
}

\begin{abstract}
The classical predator-prey equations are in nearly every differential equations text and mathematical biology text. Usually they are presented fait accompli, leaving the student to analyze them or play with a computer program. Here we show that the process of fully understanding where these equations come from and how they are derived provides numerous opportunities to teach or reinforce quantitative reasoning skills necessary to future scientists. This example is used to invoke logic, systems thinking, causal reasoning, understanding functions of one or more variables, quantities versus rates of change, proportional reasoning, unit analysis, and comparison to data.
\end{abstract}

\section{Keywords}

Quantitative reasoning, mathematical biology, science education, predator-prey model

\section{Creative Commons License}

\section{(c) (i) (9)}

This work is licensed under a Creative Commons Attribution-Noncommercial 4.0 License

\section{Cover Page Footnote}

Dorothy Wallace is a professor of mathematics at Dartmouth. She was 2000 New Hampshire CASE Professor of the Year, and the lead PI of the seminal NSF project, Mathematics Across the Curriculum. She recently finished a text in mathematical biology for first-year students, "Situated Complexity." She was a charter board member of the National Numeracy Network and is now co-editor of this journal. 


\title{
Parts Of The Whole
}

\section{A Column by $\mathrm{D}$. Wallace}

\begin{abstract}
The problem of how best to improve the numeracy of a society is a thorny one, embracing the learning process of a single student but rising in scale to include the management and alteration of an entire system of education. With the issue of quantitative literacy always in mind, this column considers various aspects of the systemic workings of education, the forces acting on classrooms, teachers and students, and mechanisms of both stasis and change. With this issue, the column pivots to thoughts from developing and teaching "Math 4: Applications of Calculus to Mathematics and Biology," which Dartmouth biology students can take as an alternative to second-semester calculus (see Rheinlander and Wallace 2011, http://dx.doi.org/10.5038/1936-4660.4.1.3 in this journal).
\end{abstract}

\section{Teaching QR in the Predator- Prey Model: Coyotes in Australia}

Numeracy is often advocated as a desirable property of good citizenship, allowing people of all backgrounds to understand the discussions of finances and resources that underlie political, social and personal decisions. In addition to that important role of numeracy we should add the role it plays in preparing future scientists to think rigorously about their assumptions, experiments, and conclusions. In this column we will look at a single example of how taking numeracy seriously can improve both experiment and theory.

In nature, most things we wish to measure are in a state of change. Although we usually take measurements at a single point in time, it's the rate of change that allows future predictions. In addition, most things we measure are part of a larger system, with many quantities changing in response to each other. Even the simplest rules imply laws of change. Newton's $F=m a$ is a static statement as written, but it implies that a change in acceleration will result in a change in force produced.

The scientific literature is full of assertions about which things cause other things to change, and by how much. Students in all scientific disciplines read these assertions constantly, understanding them as qualitative statements but perhaps not understanding that almost every assumption or assertion can be quantified, yielding quantitative conclusions that can be tested against reality. It's the business of quantitative reasoning to help students take that next step. In what follows we deconstruct the usual predator-prey equations in terms of the quantitative reasoning needed to make biological sense out of them. The skills we 
will invoke include logic, systems thinking, causal reasoning, understanding functions of one or more variables, quantities versus rates of change, proportional reasoning, unit analysis, and comparison to data. Because rates of change are involved, calculus is implicitly part of the discussion. However, no actual calculus is needed here other than the general idea of rates of change as derivatives of functions.

The predator-prey equations are derived from a scenario in which one species, the predator " $\mathrm{C}$ " (for coyote in this example), eats another species, the prey " $\mathrm{B}$ " (for bunny), in order to reproduce and survive. The population of prey " $B$ " would grow larger if the population of predator, " $C$ ", were not there. On the other hand, the population of predator, “C”, would die out if the population of prey "B" were not present. The reader may notice that in this short description we already see the act of quantification: $B$ and $C$ are now populations (variables) rather than species tags. Furthermore these populations will change, so $B$ and $C$ must become functions of time to be useful to answer simple research questions, such as: What is the long term behavior of these populations? How will populations respond if either the predator or the prey population changes suddenly? Finally, because $B$ and $C$ must change, the rates at which they do so become important. These rates can be denoted $B^{\prime}$ and $C^{\prime}$ in the language of calculus, but they have a simple meaning: change in population per unit time.

\section{Systems Thinking: If You Can't Model It, You Don't Understand It}

Clearly students understand what it means for a coyote to eat a rabbit. But that is only one of the things that has to happen in a model for populations. In the absence of coyotes, the rabbit population should grow according to some kind of rule. In the absence of rabbits, the coyote population would die. So there are three processes that need to be modeled. Before any equations are written, it helps students to create a visual model of these processes, as shown in Figure 1.

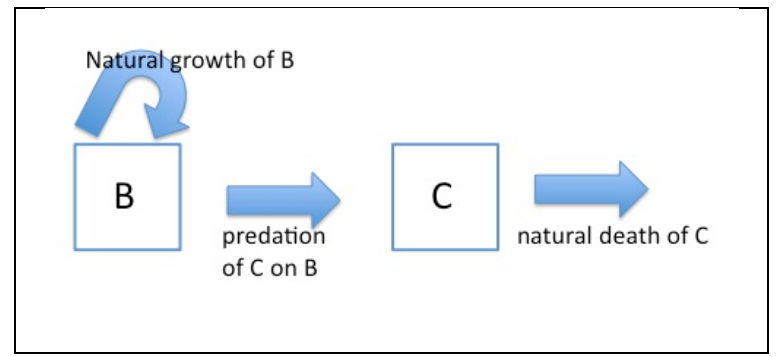

Figure 1. Getting started: Draw a diagram!

Based on my own teaching experience, a student who cannot draw this picture will not be able to construct a good expression of these processes as 
equations. Drawing a diagram is the first step in understanding complex systems. The power of the image lies in its generality: it could be rabbits and coyotes, or whales and krill, or zooplankton and phytoplankton. It describes a system that could represent multiple situations.

\section{Identifying Assumptions}

The visual model allows students to reflect on and discuss the assumptions they bring to the table. According to this image (Fig. 1), if $B$ disappears, $C$ will have only one process remaining: death. So one assumption of this visual model is that $C$ has no other food source. Similarly, $B$ appears to have only one predator. Are these reasonable assumptions? Often, the answer is yes, or almost yes. Often, the answer can be no. It depends on the organism, the biology and the ecosystem. Grappling with the ambiguities inherent in simplifying a complex problem in order to make a model is important for biology students, but equally important for mathematics students whose textbooks generally present a model as a given: this is how things work; now do some math.

The picture dictates the form the corresponding model will take. The rate of change of $B$ is equal to inputs minus outputs, each of which is shown by an arrow. The same goes for the rate of change of $C$. It is a short step from the picture to the following equations:

Rate of change of $B=$ Rate of growth of $B$ - Rate of loss of $B$ due to predation by $C$

Rate of change of $C=$ Rate of growth of $C$ due to predation on $B-$ Rate of death of $C$

This step is the first one in building a model, and it reflects basic assumptions and understanding about how the biological system works. If students can't do this, they do not understand the system they are studying in the first place.

\section{In What Units Shall We Measure B and C?}

The rate of change of $B$ is change in population of $B$ per unit time. Time units are relatively straightforward, as we can choose seconds, minutes, hours, days, weeks, years, etc. What will be the consequence of choosing minutes as units if we want to predict a population for decades? Our computer software, which we have explained to the students, will run really slowly. What will happen if we use a decade as our time unit? It's a scale that is not really suited to describing birth, death, or predation. Somehow we have to pick a time unit that makes sense in terms of the behavior of the species involved, and also in terms of the research question we would like to answer.

More complicated is the choice of units for $B$. Because we have framed the problem as "population" the naïve choice of units is "number of rabbits." This choice only makes sense in the abstract. As soon as you ask a specific question about a specific place, there is trouble. Suppose the research question is "What 
would happen to the rabbit population in Australia if coyotes were introduced to eat them?” (Ignore for a moment what might happen to the kangaroo population.) Now we are forced to wonder what number of rabbits we are talking about. All the rabbits in Australia? People may have estimated this, but nobody can actually count them. People can count rabbits in smaller regions, however. So what region might we look at? And is there a research paper that gives a data point on this question? Even a count for geographic region within Australia is probably not a count-it's an estimate which is based on samples of much smaller areas, say a square kilometer or less. Since the data we are likely to find is really population density, perhaps that is a better unit to use: number of rabbits per square kilometer, for example.

Even these units don't work well from the point of view of the coyote, however, because rabbits are not all the same size. It could take two or three small rabbits to provide the same growth benefit to a coyote as one large rabbit. It makes more sense to measure what predators eat in terms of biomass, or weight. If you find research papers measuring how much a coyote eats per day, how will the researchers report their findings? Most likely it will be reported by weight, not number of individuals. In fact, reports of consumption rates often come from the gut contents of dissected specimens, and these contents are dried before measuring. So "dry weight" is given in grams, often with a helpful conversion factor to "wet" weight of a live organism. So it really makes more sense to measure rabbit population as "kilograms of rabbit per square kilometer" than in terms of number.

More thoughts lead to the observation that large coyotes will probably eat more than small ones, for a variety of possible reasons. So it also makes sense to measure the coyote population as "kilograms of coyote per square kilometer."

At this point most students will denote both of these units as $\mathrm{kg} / \mathrm{km}^{2}$, leading to inevitable confusion later, as introducing $7 \mathrm{~kg}$ of rabbit has a different effect on the model than $7 \mathrm{~kg}$ of coyote. In any case, we may assume at this point that $B^{\prime}$ is measured in "kg of rabbit per $\mathrm{km}^{2}$ per day" and $C^{\prime}$ is measured in " $\mathrm{kg}$ of coyote per $\mathrm{km}^{2}$ per day".

Every textbook in differential equations or mathematical biology includes the predator-prey equations. Unfortunately almost none of them include any discussion of the units in which quantities and their rates of change are expressed. The absence of such a discussion misses a valuable opportunity to teach some quantitative reasoning essential to scientific research, as well as valuable skills needed to make a model relevant to a particular situation.

\section{Representing Processes as Functions}

Quantitatively literate students should be able, based on a discussion of the processes of birth, predation, and death, to identify that the natural growth of $B$ is 
a function of $B$ only, and the natural death of $C$ is a function of $C$ only. This is not as simple as it looks. Students typically want to make these terms into functions of time, second-guessing what the answer is "supposed" to look like. Biology students have seen graphs in biology texts that oscillate, for example, so they put oscillating functions of time into these terms. By the end of a thorough discussion, students should be aware that graphs of populations that they may have seen are recording certain quantities, but not the rates of change of those quantities. They should also understand that models ought to make intuitive sense, and it does not make sense for birth and death rates to depend upon the time (or date) unless external factors such as seasonality are to be taken into account. It does make sense to ask if the birth rate of $B$ might depend on available habitat or some other constraint. The rate of natural growth of $B$ will be different depending on that assumption, and the behavior of the model will also be different. These considerations all involve quantitative reasoning in the sciences, and that lesson in QR is lost if the students are just given the equations. The rest of this discussion will center on the two rates that come from the act of predation, which are even more interesting.

Students will understand that the act of predation depends on the presence of both predator and prey, so the "rate of loss of $B$ due to predation by $C$ " should be a function of both $B$ and $C$. A discussion may be required to decide that the function is independent of time. Let us call this function $F(B, C)$. At this point most textbooks would just tell you what to use for $F(B, C)$, but that would deprive us of some valuable quantitative reasoning. So let us ask the students a few questions.

1. What should $F$ be if $C$ is not present?

2. What should $F$ be if $B$ is not present?

3. If $B$ is held constant (not zero) and $C$ is increased, what should $F$ do?

4. If $C$ is held constant (not zero) and $B$ is increased, what should $F$ do?

It doesn't take students long to say that

1. If no predator or prey is present, $F$ should be zero.

2. If either predator or prey rises, the rate of predation will probably go up.

Students invited to invent examples of $F$ that do these four things will come up with a variety of expressions. They can all be judged against these four criteria. When it comes time to decide on an expression, the classical form, $F(B, C)=k * B * C$, will be acceptable as one option that meets the criteria. 


\section{Unit Analysis}

At this point it is important to revisit the discussion of units to figure out what units the constant $k$ will have to be. We have decided to measure the rate of change of $B$ in $\mathrm{kg}$ of rabbit per $\mathrm{km}^{2}$ per day, $B$ in $\mathrm{kg}$ of rabbit per $\mathrm{km}^{2}$, and $C$ in $\mathrm{kg}$ of coyote per $\mathrm{km}^{2}$. Abbreviating "kg of rabbit" as $\mathrm{kgB}$ and "kg of C" as $\mathrm{kgC}$, we can move on and insist the units agree. This gives

$\mathrm{kgB} /\left(\mathrm{km}^{2} \cdot\right.$ day $)=k * \mathrm{kgB} / \mathrm{km}^{2} * \mathrm{kgC} / \mathrm{km}^{2}$

and forces $k$ to be in units of $\left(\mathrm{kgC} / \mathrm{km}^{2}\right)^{-1}$ per day. These units are not easy to describe, but they tell us what proportion of the biomass of $B$ is eaten per $\operatorname{kg}$ of $C$ in a day (within a square kilometer).

The act of figuring out what this means can potentially bring the model closer to observational data than any other part of the modeling process. Suppose we find a paper that measures a single coyote fenced in with 40 rabbits an area of $1 / 10$ of a square kilometer (about 25 acres). Suppose the coyote weighs $20 \mathrm{~kg}$ and the rabbits (combined) weigh $200 \mathrm{~kg}$. Suppose further that the coyote was observed to eat $0.5 \mathrm{~kg}$ of rabbit in a day. We then have:

1. loss of rabbits due to predation $=\frac{0.5 \mathrm{kgB}}{\left(0.1 \mathrm{~km}^{2}\right)(\text { day })}$

2. $B=200 \mathrm{kgB} / 0.1 \mathrm{~km}^{2}$

3. $C=20 \mathrm{kgC} / 0.1 \mathrm{~km}^{2}$,

from which, using $B^{\prime}=k B C$,

$\frac{0.5 \mathrm{kgB}}{\left(0.1 \mathrm{~km}^{2}\right)(\text { day })}=k\left(\frac{200 \mathrm{kgB}}{0.1 \mathrm{~km}^{2}}\right)\left(\frac{20 \mathrm{kgC}}{0.1 \mathrm{~km}^{2}}\right)$. And so, $=\left(\frac{0.5}{0.1}\right)\left(\frac{0.1}{200}\right)\left(\frac{0.1}{20}\right) \frac{\left(\mathrm{kgC} / \mathrm{km}^{2}\right)^{-1}}{\mathrm{day}}$.

What about the gain of coyote biomass due to predation? Clearly it is also a function of both $B$ and $C$, but is it the same function, $F$ ? If invited to think about many examples the students will quickly realize that, although the two functions are proportional, there should be more prey lost than predator gained. Even the students most unaware of the natural world can answer these questions:

1. If you eat a pound of food, do you gain a pound of weight?

2. If you eat a pound of food, do you make a pound of offspring?

3. Are these numbers even close?

Somewhere there are data on how much coyotes eat per day and how fast they grow and reproduce. This data can be wrangled as in the last example to estimate the relative size of the two terms. 


\section{Proportional Reasoning}

The term for loss of $B$ due to predation $(k * B * C)$ is a double proportionality. That means that if you double the amount of predators, twice as much prey will get eaten. If you double the amount of prey, twice as much prey will get eaten.

Suppose there is exactly one coyote. If you double the amount of rabbits, the $B$ factor says the coyote will eat twice as much. If you multiply the number of rabbits by 100, this poor coyote will have to eat 100 times as much prey, according to the formula. Understanding this is an exercise in proportional reasoning that ought to lead to a discussion of the limitations of models. If there are not too few or too many of the predator or prey, the model may be a reasonable description. But it fails at some extremes.

If, at this point, students are invited to develop a form of the function $F$ that works better at extreme values of populations, they will learn a lot about functional forms. And if they find one that works, they will have to rethink what the constant " $k$ " must mean and how to measure it.

\section{Why Don't We Teach This Way?}

The present form of mathematics classes comes from emphasizing knowledge and skills valued in that discipline. Similarly, the present form of biology classes comes from emphasizing facts, procedures, and experimental inquiry valued in that discipline. Teaching the type of material described here requires courses that begin with the intention to span both disciplines and put a priority on the ability of the learner to do so. Mathematics could be of far greater use to scientists than it currently is with one or more specific courses aimed at STEM majors other than physics and engineering. A necessary part of the bridge between math and other subjects lies in the domain of quantitative reasoning, which can serve scientists at all stages of their careers. 